\title{
A Non-Stationary MIMO Channel Model for High-Speed Train Communication Systems
}

\author{
Ammar Ghazal ${ }^{1}$, Cheng-Xiang Wang ${ }^{1}$, Harald Haas ${ }^{2}$, Mark Beach ${ }^{3}$, Xiaofeng $\mathrm{Lu}^{4}$, Dongfeng Yuan ${ }^{5}$, \\ and Xiaohu $\mathrm{Ge}^{6}$ \\ ${ }^{1}$ Joint Research Institute for Signal and Image Processing, Heriot-Watt University, Edinburgh EH14 4AS, UK. \\ ${ }^{2}$ Joint Research Institute for Signal and Image Processing, University of Edinburgh, Edinburgh EH9 3JL, UK. \\ ${ }^{3}$ Department of Electrical \& Electronic Engineering, University of Bristol, Bristol BS8 1UB, UK. \\ ${ }^{4}$ Huawei Technologies Co., Ltd., Xi'an, 717705, China. \\ ${ }^{5}$ School of Information Science and Engineering, Shandong University, Jinan, 250100, China. \\ ${ }^{6}$ Department of Electronics and Information Engineering, Huazhong University of Science and Technology, Wuhan 430074, Hubei, China. \\ Email: \{ag289, cheng-xiang.wang\}@hw.ac.uk, h.haas@ed.ac.uk, m.a.beach@bristol.ac.uk, stan.x.lu@huawei.com,dfyuan@sdu.edu.cn, \\ xhge@mail.hust.edu.cn
}

\begin{abstract}
This paper proposes a non-stationary wideband geometry-based stochastic model (GBSM) for multiple-input multiple-output (MIMO) high-speed train (HST) channels. The proposed model has the ability to investigate the non-stationarity of HST environment caused by the high speed movement of the receiver. Based on the proposed model, the space-time-frequency (STF) correlation function (CF) and STF local scattering function (LSF) are derived for different taps. Numerical results show the non-stationarity of the proposed channel model.
\end{abstract}

\section{INTRODUCTION}

Due to the growing demand for broadband services in the area of passenger transport, the implementation of wireless communication systems in HSTs has attracted great interest from communication companies. The existing wireless communication system for railways, Global System for Mobile Communication Railway (GSM-R), cannot meet the requirements for high transmission rates to satisfy the demands of current broadband users, besides the fact that GSM-R is used for train control rather than providing communications for train passengers. Using current candidates for the fourth generation (4G) wireless systems such as IMT-Advanced (IMT-A) [1] and Long Term Evolution-Advanced (LTE-A) [2] needs to overcome various challenges that face these systems in high mobility environments. Challenges like fast handover [3], high penetration losses [4], and limited visibility in tunnels [5], besides the varying Doppler, delay, and angular spreads [6] due to the high-speed link between transmitters and receivers, need to be addressed. Therefore, investigations into radio propagation characteristics for wireless communication systems that are capable of providing broadband communications in HSTs have been initiated.

The key features of wireless channels in HST communication systems are time-varying and non-stationarity. Although several measurement campaigns have been conducted for highvelocity scenarios [7]-[11] but they mainly focused on large scale parameters like path loss and the root mean square (RMS) delay spread and ignored other propagation characteristics. Table 1 classifies some of the HST measurement cam- paigns according to carrier frequencies, bandwidth, antennas, measured channel and channel statistics.

On the other hand, channel models in the literature have also failed to characterize different propagation parameters of similar scenarios. 3GPP defines a channel model for highspeed train tests in LTE-A. However, this model is a nonfading propagation channel model and consists of one ray only. It mainly concentrates on studying the impact of Doppler effect on system performance while ignoring other channel characteristics. WINNER-II [12] and IMT-A channel models also consider high-speed scenarios in their rural macro-cell (RMa) and moving networks scenarios, respectively, where the MS antenna speed is in the range of $0-350 \mathrm{~km} / \mathrm{h}$. The proposed scenario considers dedicated mobile relay stations (MRSs) deployed on the surface of the train to improve the coverage on the carriages by extending the coverage of the outdoor base stations (BSs). Therefore, two types of channels can be distinguished, i.e., BS-MRS and MRS-mobile station (MS) channels. IMT-A considers that the BS-MRS channel satisfies the assumption of wide-sense stationary uncorrelated scattering (WSSUS) neglecting the non-stationarity of this channel. It also considers an MRS-MS channel as an indoor one ignoring the effect of the surrounding environment. As indicated in [8], waves re-entering the carriage from outside of the train can be dominant in certain cases. It also showed that the propagation loss in a train carriage can be less than the free space one due to the waveguide effect in a closed space environment like trains. In [6] and [13], the propagation channels between HSTs and fixed BSs were modeled using the ray-tracing method which incorporates a detailed simulation of the actual physical wave propagation process based on Maxwell equations. The authors presented two-dimensional (2D) ray-tracing [6] and three-dimensional (3D) ray-tracing [13] channel models to investigate the effects of different antenna types on the channel impulse response and how to reduce the inter-carrier interference (ICI) and inter-symbol interference (ISI). However, the implementation of ray-tracing models always requires extensive computational resources 
[14]. The aforementioned examples have motivated us to investigate more thoroughly the parameters that distinguish the HST scenario from other propagation scenarios and to design an accurate channel model that is able to mimic the key parameters of similar radio environments.

The rest of this paper is organized as follows. A GBSM for MIMO HST channels is proposed in Section II. In Section III, the STF CF and LSF of the GBSM are derived. The numerical results and the analysis are presented in Section IV. Finally, the conclusions are drawn in Section V.

\section{THE GBSM}

We consider a MIMO HST communication system with $S$ transmit and $U$ receive omni-directional antenna elements. The BS is considered to be located on the track-side $\left(D_{\min }=2 \mathrm{~m}\right)$ as in [1]. Both the BS and MRS are equipped with multiple antennas at equivalent heights as shown in Fig. 1. Fig. 2 illustrates the geometry of the proposed GBSM, which consists of multiple confocal ellipses with single-bounced rays and the line-of-sight (LoS) component [15]. For clarity purposes, in this figure, we use a $2 \times 2$ MIMO channel model as an example. The multiple confocal ellipses model with the BS and MRS located at the foci represents the tapped delay line (TDL) structure and has $N$ effective scatterers on the $i$ th ellipse (i.e., $i$ th tap), where $i=1,2, \ldots, I$ and $I$ is the total number of ellipses or taps. The semi-major axis of the $i$ th ellipse and the $n_{i}$ th $\left(n_{i}=1, \ldots, N\right)$ effective scatterer are denoted by $a_{i}$ and $s^{\left(n_{i}\right)}$, respectively. The initial distance between the BS and MRS is $D_{s}=2 f$ with $f$ representing a half of the distance between the two focal points of ellipses. The antenna element spacings at the BS and MRS are denoted by $\delta_{T}$ and $\delta_{R}$, respectively. The multi-element antenna tilt angles are denoted by $\beta_{T}$ and $\beta_{R}$. The MRS moves with the train with speed $v_{R}$ in the direction determined by the angles of motion $\gamma_{R}$. The angle-of-arrival (AoA) of the wave traveling from an effective scatterer $s^{\left(n_{i}\right)}$ to the MRS is denoted by $\phi_{R}^{\left(n_{i}\right)}$. The angle-ofdeparture (AoD) of the wave that impinges on the effective scatterer $s^{\left(n_{i}\right)}$ is denoted by $\phi_{T}^{\left(n_{i}\right)}$, while $\phi_{T_{p}}^{\mathrm{LoS}}$ denotes the AoA of a LoS path.

Based on the TDL concept, the complex impulse response between the $p$ th $(p=1, \ldots, S)$ element of the BS, $T_{p}$, and the $q$ th $(q=1, \ldots, U)$ element of the MRS, $R_{q}$, can be expressed as $h_{p q}\left(t, \tau^{\prime}\right)=\sum_{i=1}^{I} h_{i, p q}(t) \delta\left(\tau^{\prime}-\tau_{i}^{\prime}\right)$ where $h_{i, p q}(t)$ and $\tau_{i}^{\prime}$ denote the complex time-variant tap coefficient and the discrete propagation delay of the $i$ th tap, respectively. From the above GBSM, the complex tap coefficient for the first tap of the $T_{p}-R_{q}$ link is a superposition of the LoS component and single-bounced components, and can be expressed as

$$
h_{1, p q}(t)=h_{1, p q}^{\mathrm{LoS}}(t)+h_{1, p q}^{\mathrm{SB}}(t)
$$

where

$$
\begin{aligned}
h_{1, p q}^{\mathrm{LoS}}(t)=\sqrt{\frac{K_{p q}}{K_{p q}+1}} e^{-j 2 \pi f_{c} \tau_{p q}(t)} & \times e^{j\left[2 \pi f_{\max } t \cos \left(\phi_{T_{p}}^{\mathrm{Los}}(t)-\gamma_{R}\right)\right]}
\end{aligned}
$$

$$
\begin{aligned}
& \text { and } \\
& \begin{aligned}
h_{1, p q}^{\mathrm{SB}}(t)=\sqrt{\frac{1}{K_{p q}+1}} \lim _{N \rightarrow \infty} \sum_{n_{1}=1}^{N} \frac{1}{\sqrt{N}} e^{j\left(\psi_{n_{1}}-2 \pi f_{c} \tau_{p q, n_{1}}(t)\right)} \\
\times e^{j\left[2 \pi f_{\max } t \cos \left(\phi_{R}^{\left(n_{1}\right)}(t)-\gamma_{R}\right)\right]} .
\end{aligned}
\end{aligned}
$$

The complex tap coefficient for other taps $(1<i \leq I)$ of the $T_{p}-R_{q}$ link is a sum of single-bounced components only and can be expressed as

$$
\begin{aligned}
h_{i, p q}(t)= & h_{i, p q}^{\mathrm{SB}}(t)=\lim _{N \rightarrow \infty} \sum_{n_{i}=1}^{N} \frac{1}{\sqrt{N}} e^{j\left(\psi_{n_{i}}-2 \pi f_{c} \tau_{p q, n_{i}}(t)\right)} \\
& \times e^{j\left[2 \pi f_{\max } t \cos \left(\phi_{R}^{\left(n_{i}\right)}(t)-\gamma_{R}\right)\right]}, \quad 1<i \leq I
\end{aligned}
$$

In (2) and (3), the total power for each tap is normalized to $1, \tau_{p q}(t)=\varepsilon_{p q}(t) / c$ and $\tau_{p q, n_{i}}(t)=\left(\varepsilon_{p n_{i}}(t)+\varepsilon_{n_{i} q}(t)\right) / c$ $(i=1,2, \ldots, I)$ are the travel times of the waves through the link $T_{p}-R_{q}$ and $T_{p}-s^{\left(n_{i}\right)}-R_{q}$, respectively, as shown in Fig. 2. Here, $c$ represents the speed of light and the symbol $K_{p q}$ designates the Ricean factor. The phases $\psi_{n_{1}}$ and $\psi_{n_{i}}$ are independent and identically distributed (i.i.d.) random variables with uniform distributions over $[-\pi, \pi)$ and $f_{\max }$ is the maximum Doppler frequency with respect to the MRS.

From Fig. 2 and based on the law of cosines, we have

$$
\begin{aligned}
& \varepsilon_{p q}(t) \approx\left(D_{s}-v t\right)-k_{p} \delta_{T} \cos \beta_{T}-k_{q} \delta_{R} \cos \left(\phi_{T_{p}}^{\mathrm{LoS}}(t)-\beta_{R}\right) \\
& \varepsilon_{p n_{i}}(t) \approx \xi_{T}^{\left(n_{i}\right)}(t)-k_{p} \delta_{T} \cos \left(\phi_{T}^{\left(n_{i}\right)}(t)-\beta_{T}\right) \\
& \varepsilon_{n_{i} q}(t) \approx \xi_{R}^{\left(n_{i}\right)}(t)-k_{q} \delta_{R} \cos \left(\phi_{R}^{\left(n_{i}\right)}(t)-\beta_{R}\right)
\end{aligned}
$$

where $k_{p}=\left(M_{T}-2 p+1\right) / 2, k_{q}=\left(M_{R}-2 q+1\right) / 2, \xi_{T}^{\left(n_{i}\right)}(t)=$ $\left(a_{i}^{2}(t)+f^{2}(t)+2 a_{i}(t) f(t) \cos \phi_{R}^{\left(n_{i}\right)}(t)\right) /\left(a_{i}(t)+f(t) \cos \phi_{R}^{\left(n_{i}\right)}(t)\right)$, and $\xi_{R}^{\left(n_{i}\right)}(t)=b_{i}^{2}(t) /\left(a_{i}(t)+f(t) \cos \phi_{R}^{\left(n_{i}\right)}(t)\right)$ with $f(t)=f-v_{R} t$. Here, $b_{i}(t)$ denotes the time-varying semi-minor axis of the $i$ th ellipse and is defined by $b_{i}(t)=\sqrt{a_{i}^{2}(t)-D_{s}^{2}(t)}$, where $a_{i}(t)=a_{i}-v_{R} t$ and $D_{s}(t)=D_{s}-v_{R} t$. The time-variant LoS AoA $\phi_{T_{p}}^{\text {LoS }}$ can be expressed as [2]

$$
\phi_{T_{p}}^{\operatorname{LoS}}(t)=\left\{\begin{array}{lr}
\arccos \left(\frac{D_{s}-v_{R} t}{\sqrt{D_{\min }^{2}+\left(D_{s}-v_{R} t\right)^{2}}}\right), & 0 \leq t \leq \frac{2 D_{s}}{v_{R}} \\
\arccos \left(\frac{-1.5 D_{s}+v_{R} t}{\sqrt{D_{\min }^{2}+\left(-1.5 D_{s}+v_{R} t\right)^{2}}}\right), & \frac{2 D_{s}}{v_{R}}<t \leq \frac{4 D_{s}}{v_{R}} \\
\phi_{T_{p}}^{\operatorname{Los}}\left(t \bmod \left(\frac{4 D_{s}}{v_{R}}\right)\right), & t>\frac{4 D_{s}}{v_{R}} .
\end{array}\right.
$$

Note that the $\operatorname{AoD} \phi_{T}^{\left(n_{i}\right)}$ and $\operatorname{AoA} \phi_{R}^{\left(n_{i}\right)}$ are interdependent for single-bounced rays. The relationship between the AoD and AoA for multiple confocal ellipses model can be expressed as [16]

$$
\begin{gathered}
\sin \phi_{T}^{\left(n_{i}\right)}(t)=\frac{b_{i}^{2}(t) \sin \phi_{R}^{\left(n_{i}\right)}(t)}{a_{i}^{2}(t)+f^{2}(t)+2 a_{i}(t) f(t) \cos \phi_{R}^{\left(n_{i}\right)}(t)} \\
\cos \phi_{T}^{\left(n_{i}\right)}(t)=\frac{2 a_{i}(t) f(t)+\left(a_{i}^{2}(t)+f^{2}(t)\right) \cos \phi_{R}^{\left(n_{i}\right)}(t)}{a_{i}^{2}(t)+f^{2}(t)+2 a_{i}(t) f(t) \cos \phi_{R}^{\left(n_{i}\right)}(t)}
\end{gathered}
$$


Since the numbers of effective scatterers are assumed to be infinite, i.e., $N \rightarrow \infty$, the proposed model is actually a mathematical reference model. Due to the infinite complexity, a reference model cannot be implemented in practice. A reference model can be used for theoretical analysis and design of a communication system, and as a starting point to design a reliable simulation model that has the reasonable complexity, i.e., with finite values of $N$. Here, we use the von Mises PDF defined as $f(\phi) \triangleq \exp [k \cos (\phi-\mu)] /\left[2 \pi I_{0}(k)\right][16]$, where $\phi \in[-\pi, \pi), I_{0}(\cdot)$ is the zeroth-order modified Bessel function of the first kind, $\mu \in[-\pi, \pi)$ accounts for the mean angular value of the angle $\phi$, and $k(k \geq 0)$ is a real-valued parameter that controls the spread of $\phi$. For the continuous AoAs $\phi_{R}^{(i)} \in[-\pi, \pi)$ and AoDs $\phi_{T}^{(i)} \in[-\pi, \pi)$, the relevant parameters in the von Mises PDF are $\mu_{R}^{(i)}$ and $k_{R}^{(i)}$ and $\mu_{T}^{(i)}$ and $k_{T}^{(i)}$, respectively. In case of isotropic scattering, $k=0$, the von Mises distribution reduces to the uniform distribution $f(\phi)=1 /(2 \pi)$.

\section{Characterization of The HSt Channel Model}

Unlike non-stationary channels [17], characterization of WSSUS channels has been investigated thoroughly in the literature [18], [19]. For the proposed non-stationary HST GBSM, we can obtain the corresponding time-varying transfer function by taking the Fourier transformation of the complex impulse response $h_{p q}\left(t, \tau^{\prime}\right)$ in terms of $\tau^{\prime}$, i.e.,

$$
L_{H_{p q}}(t, f)=\int h_{p q}\left(t, \tau^{\prime}\right) e^{-j 2 \pi f \tau^{\prime}} \mathrm{d} \tau^{\prime}
$$

which essentially is a non-stationary process for HST scenarios. The channel correlations/nonstationarties that have been described in [20] can be extended to an STF CF and expressed as

$R_{L_{p q} L_{p^{\prime} q^{\prime}}}(t, f ; \Delta t, \Delta f)=\mathbf{E}\left[L_{H_{p q}}(t, f+\Delta f) L_{H_{p^{\prime} q^{\prime}}}^{*}(t-\Delta t, f)\right]$

where $(\cdot)^{*}$ denotes the complex conjugate operation, $\mathbf{E}[\cdot]$ designates the statistical expectation operator, $\Delta t$ and $\Delta f$ denote the time lag and frequency lag, respectively. The LSF describes the mean power of the effective scatterers that cause delay-Doppler shifts $(\tau, \nu)$ at time $t$ and frequency $f$. It is defined as a space-time-frequency dependant scattering function of the non-stationary process $L_{H_{p q}}(t, f)$, i.e.,

$$
\begin{aligned}
C_{H_{p q} H_{p^{\prime} q^{\prime}}}(t, f, \tau, \nu)=\iint & R_{L_{p q} L_{p^{\prime} q^{\prime}}}(t, f ; \Delta t, \Delta f) \\
& \times e^{-j 2 \pi(\nu \Delta t-\tau \Delta f)} \mathrm{d} \Delta t \mathrm{~d} \Delta f .
\end{aligned}
$$

By applying the corresponding von Mises distribution, and the equality $\int_{-\pi}^{\pi} \exp (a \sin c+b \cos c) \mathrm{d} c=2 \pi I_{0}\left(\sqrt{a^{2}+b^{2}}\right)$, we can obtain $R_{L}(t, f ; \Delta t, \Delta f)$ of the LoS and single-bounced components of different taps as follows.

1) In the case of the LoS component

$$
\begin{aligned}
L_{H_{1, p q}}^{\mathrm{LoS}}(t, f)= & \sqrt{\frac{K_{p q}}{K_{p q}+1}} e^{-j 2 \pi f_{c} \tau_{p q}(t)} \\
& \times e^{j\left[2 \pi f_{\max } t \cos \left(\phi_{T_{p}}^{\mathrm{LoS}}(t)-\gamma_{R}\right)\right]} e^{-j 2 \pi f \tau_{1}^{\prime}}
\end{aligned}
$$

$$
R_{L_{1, p q}^{\mathrm{Los}} L_{1, p^{\prime} q^{\prime}}^{\mathrm{Los}}}(t, f ; \Delta t, \Delta f)=K^{\prime} e^{j 2 \pi\left(G+\Delta t W(t)-\Delta f \tau_{1}^{\prime}\right)}
$$

where $G=P \cos \beta_{T}-Q \cos \beta_{R}$, with $P=\left(p^{\prime}-p\right) \delta_{T} / \lambda, Q=$ $\left(q^{\prime}-q\right) \delta_{R} / \lambda, W(t)=f_{\max } \cos \left(\phi_{T_{p}}^{\operatorname{LoS}}(t)-\gamma_{R}\right)$, and $K^{\prime}=$ $\sqrt{\frac{K_{p q} K_{p^{\prime} q^{\prime}}}{\left(K_{p q}+1\right)\left(K_{p^{\prime} q^{\prime}}+1\right)}}$.

2) In terms of the single-bounced components

$$
\begin{gathered}
L_{H_{i, p q}}^{\mathrm{SB}}(t, f)=\sqrt{\frac{1}{K_{p q}+1}} \lim _{N \rightarrow \infty} \sum_{n_{i}=1}^{N} \frac{1}{\sqrt{N}} e^{j\left(\psi_{n_{i}}-2 \pi f_{c} \tau_{p q, n_{i}}(t)\right)} \\
\times e^{j\left[2 \pi f_{\max } t \cos \left(\phi_{R}^{\left(n_{i}\right)}(t)-\gamma_{R}\right)\right]} e^{-j 2 \pi f \tau_{i}^{\prime}} \\
R_{L_{i, p q}^{\mathrm{SB}} L_{i, p^{\prime} q^{\prime}}^{\mathrm{SB}}(t, f ; \Delta t, \Delta f)}=\frac{1}{2 \pi I_{0}\left(k_{R}^{(i)}\right) U} \\
\int_{-\pi}^{\pi} e^{k_{R}^{(i)} \cos \left(\phi_{R}^{(i)}-\mu_{R}^{(i)}\right)} e^{j 2 \pi\left[P \cos \left(\phi_{T}^{(i)}-\beta_{T}\right)+Q \cos \left(\phi_{R}^{(i)}-\beta_{R}\right)\right]} \\
\times e^{j 2 \pi \Delta t f_{\max } t \cos \left(\phi_{R}^{(i)}-\gamma_{R}\right)} e^{-j 2 \pi \Delta f \tau_{i}^{\prime}} d \phi_{R}^{(i)}
\end{gathered}
$$

where $K_{p q}$ and $U=\sqrt{\left(K_{p q}+1\right)\left(K_{p^{\prime} q^{\prime}}+1\right)}$ only appear for the first tap. Substituting (11) and (13) into (9), we can obtain the corresponding LSFs.

1) In the case of the LoS component

$$
\begin{array}{r}
C_{H}^{\mathrm{LoS}}(t, f, \tau, \nu)=\iint R_{L_{p q}^{\mathrm{Los}} L_{p^{\prime} q^{\prime}}^{\mathrm{Los}}(t, f ; \Delta t, \Delta f)} \\
\times e^{-j 2 \pi(\nu \Delta t-\tau \Delta f)} \mathrm{d} \Delta t \mathrm{~d} \Delta f \\
=\iint K^{\prime} e^{j 2 \pi\left(G+(W(t)-\nu) \Delta t+\left(\tau-\tau_{1}^{\prime}\right) \Delta f\right)} \mathrm{d} \Delta t \mathrm{~d} \Delta f .
\end{array}
$$

2) In the case of the single-bounced components

$$
C_{H_{i}}^{\mathrm{SB}}(t, f, \tau, \nu)=
$$

$$
\iint R_{L_{i, p q}^{\mathrm{SB}} L_{i, p^{\prime} q^{\prime}}^{\mathrm{SB}}}(t, f ; \Delta t, \Delta f) e^{-j 2 \pi(\nu \Delta t-\tau \Delta f)} \mathrm{d} \Delta t \mathrm{~d} \Delta f
$$

The integrals in (14) and (15) will be evaluated numerically.

\section{Numerical Results And Analysis}

In this section, the analytical expressions provided in the previous section are evaluated numerically. From the moving networks scenario in [12] that was designed based on some measurements in HST environments, we have chosen the following parameters: the LoS Ricean factor $K_{p q}=K_{p^{\prime} q^{\prime}}$ $=6 \mathrm{~dB}$, taps delay for the first and second tap are $\tau_{1}^{\prime}=$ 0 and $\tau_{2}^{\prime}=50 \mathrm{~ns}$, respectively, $f_{c}=6 \mathrm{GHz}, v=360$ $\mathrm{km} / \mathrm{h}, f_{\max }=2 \mathrm{kHz}, D_{s}=500 \mathrm{~m}, a_{1}=\frac{D_{s}}{2}+150 \mathrm{~m}$, $a_{2}=\frac{D_{s}}{2}+180 \mathrm{~m}, \gamma_{R}=0$ and we are using a uniform antenna array with $M_{R}=M_{T}=2$ and $\beta_{R}=\beta_{T}=\pi / 2$. Figs. 3 and 4 show the absolute values of the theoretical STF CFs $R_{L_{p q} L_{p^{\prime} q^{\prime}}}(t, f ; \Delta t, \Delta f)$ of the proposed HST model for the first tap and second tap, respectively, when considering an isotropic environment $k_{R}=0$ and adopting a BS antenna elements spacing $\delta_{T}=\lambda$. We can see that the STF CF changes for different values of $t$, which is due to the non-stationarity 
of the channel model. Fig. 3 shows higher correlations than Fig. 4 proving that the LoS component, which is dominant in HST scenarios [1], [2] and [12], increases the correlation between different antenna elements and as a result it reduces the benefit of using MIMO techniques in such environments.

The absolute values of the normalized STF LSF of the second tap of the channel model $C_{H_{2}}^{\mathrm{SB}}(t, f, \tau, \nu)$ are shown in Fig. 5. It can be seen from this figure how the power of the effective scatters varies with time $t$. It can also be noticed that the power of components with zero Doppler frequency is higher than the rest of the components for all time instants $t$.

The normalized STF LSF of the proposed HST model for different taps with delays $\tau_{i}$ at a certain time instant and with the assumption of no correlation between different taps is reduced to Doppler power spectrum density (PSD) function which is illustrated in Fig. 6. From this figure, we can see that different taps have the same classic Doppler spectrum at the time instant $t=0$.

\section{Conclusions}

Non-stationary channel models are increasingly important for designing and evaluating different proposed solutions for future HST communication systems. In this paper, we have proposed a non-stationary wideband MIMO HST GBSM that takes into account the LoS component and single-bounced rays. The proposed channel model enables us to study the time-varying channel statistics. From this model, we have derived the STF CFs and STF LSFs for different taps. Numerical results have proved that the stationary assumption is violated for HST channels.

\section{ACKNOWLEDGEMENTS}

A. Ghazal acknowledges the sponsorship of his work by Damascus University. The authors would like to acknowledge the support from the RCUK for the UK-China Science Bridges Project: R\&D on (B) 4G Wireless Mobile Communications. A. Ghazal, C.-X. Wang, and $\mathrm{H}$. Haas would also like to acknowledge the support from the Scottish Funding Council for the Joint Research Institute in Signal and Image Processing between the University of Edinburgh and Heriot-Watt University, as part of the Edinburgh Research Partnership in Engineering and Mathematics (ERPem). C.-X. Wang also thanks the support from Huawei Technologies and the Opening Project of the Key Laboratory of Cognitive Radio and Information Processing (Guilin University of Electronic Technology), Ministry of Education (Grant No.: 2011KF01). X. Ge acknowledges the support from the National Natural Science Foundation of China (NSFC) (Grant No.: 60872007), National 863 High Technology Program of China (Grant No.: 2009AA01Z239), the Ministry of Science and Technology (MOST), China, International Science and Technology Collaboration Program (Grant No.: 0903) and Hubei Provincial Science and Technology Department (Grant No.: 2011BFA004).

\section{REFERENCES}

[1] ITU-R M.2135-1, "Guidelines for Evaluation of Radio Interface Technologies for IMT-Advanced," Geneva, Switzerland, Rep. ITU-R M.21351, Dec. 2009

[2] 3GPP, TS36.101, V10.2.1, "3rd Generation Partnership Project; Technical Specification Group Radio Access Network; Evolved Universal Terrestrial Radio Access (E-UTRA); User Equipment (UE) radio transmission and reception (Release 10),” Apr. 2011.
[3] H. Wu, Y. Gu and Z. Zhong, "Research on the fast algorithm for GSMR switching for high-speed railway," Journal of Railway Engineering Society, pp. 92-98, Jan. 2009.

[4] Y. Zhou, Z. Pan, J. Hu, J. Shi, and X. Mo, "Broadband wireless communications on high speed trains," in Proc. IEEE WOCC'11, New Jersey, USA, Apr. 2011, pp.1-6.

[5] C. Briso-Rodriguez, J.M. Cruz, and J.I. Alonso, "Measurements and modeling of distributed antenna systems in railway tunnels," IEEE Trans. Vehicular Technology, vol. 56, no. 5, pp.2870-2879, Sept. 2007.

[6] S. Knorzer, T. Fugen and W. Wiesbeck, "Ray-tracing for mobile communications," in Proc. of Wave Propagation in Communication, Microwave Systems and Navigation, Chemnitz, Germany, 2007.

[7] W. Dong, G. Liu, L. Yu, H. Ding and J. Zhang, "Channel properties of indoor part for high-speed train based on wideband channel measurement," in Proc. IEEE CHINACOM'10, Beijing, China, Aug. 2010, pp. 1-4.

[8] N. Kita, T. Ito, S. Yokoyama, Ming-Chien Tseng, Y. Sagawa, M. Ogasawara and M. Nakatsugawa, "Experimental study of propagation characteristics for wireless communications in high-speed train cars," in Proc. IEEE EuCAP'09, Berlin, Germany, Mar. 2009, pp. 897-901.

[9] P. Aikio, R. Gruber and P. Vainikainen, "Wideband radio channel measurements for train tunnels," in Proc. IEEE VTC'98, Ottawa, Canada, May 1998, pp. 460-464.

[10] R. Parviainen, P. Kyosti, Y. Hsieh, P. Ting and J. Chiou, "Results of high speed train channel measurements," in COST 2100 TD'08, Lille, France, Oct. 2008.

[11] R. He, Z. Zhong, and B. Ai, "Path loss measurements and analysis for high-speed railway viaduct scene," in Proc. IWCMC'10, Caen, France, June 2010, pp. 266-270.

[12] P. Kyösti, et al., "WINNER II channel models," IST-4-027756, WINNER II D1.1.2, v1.2, Apr. 2008.

[13] S. Knorzer, M. A. Baldauf, T. Fugen, and W. Wiesbeck, "Channel analysis for an OFDM-MISO train communications system using different antennas," in Proc. IEEE VTC'07-Fall, Baltimore, USA, Oct. 2007, pp. 809-813.

[14] K. Pahlavan and A. Levesquec, Wireless Information Networks. 2nd ed. NewYork: Wiley, 2005.

[15] M. Pätzold and B.O Hogstad, "A wideband MIMO channel model derived from the geometric elliptical scattering model," in Proc. IEEE ISWCS'06, Valencia, Spain, Sept. 2006, pp. 138-143.

[16] X. Cheng, C.-X. Wang, D. I Laurenson, S. Salous, and A. V. Vasilakos, "An adaptive geometry-based stochastic model for non-isotropic MIMO mobile-to-mobile channels," IEEE Trans. Wireless Commun., vol. 8, no. 9, Sept. 2009, pp. 4824-4835.

[17] C.-X. Wang, X. Cheng, and D. I. Laurenson, "Vehicle-to-vehicle channel modeling and measurements: recent advances and future challenges," IEEE Commun. Mag., vol. 47, no. 11, pp. 96-103, Nov. 2009.

[18] C.-X. Wang, M. Pätzold, and Q. Yao, "Stochastic modeling and simulation of frequency correlated wideband fading channels," IEEE Trans. Vehicular Technology, vol. 56, no. 3, pp. 1050-1063, May 2007.

[19] C.-X. Wang, X. Hong, H. Wu, and W. Xu,"Spatial temporal correlation properties of the 3GPP spatial channel model and the Kronecker MIMO channel model," EURASIP J. Wireless Commun. and Networking, vol. 2007, Article ID 39871, 9 pages, 2007. doi:10.1155/2007/39871.

[20] G. Matz, "On non-WSSUS wireless fading channels," IEEE Trans. on Wireless Commun., vol. 4, no. 5, pp. 2465-2478, Sept. 2005.

TABLE I

HST CHANNEL MEASUREMENTS.

\begin{tabular}{|l|l|l|l|l|l|}
\hline Meas. & Channel & $\begin{array}{l}\text { Carrier } \\
\text { Frequency }\end{array}$ & $\begin{array}{l}\text { Band- } \\
\text { width }\end{array}$ & Antenna & $\begin{array}{l}\text { Channel } \\
\text { Statistics }\end{array}$ \\
\hline$[7]$ & Indoor & $2.35 \mathrm{GHz}$ & $100 \mathrm{MHz}$ & SISO & PL, DS, $K$ \\
\hline$[8]$ & $\begin{array}{l}\text { Inter-car/ } \\
\text { Intra-car }\end{array}$ & $\begin{array}{l}5.2 \mathrm{GHz} / \\
2.2 \mathrm{GHz}\end{array}$ & $20 \mathrm{MHz}$ & SISO & PL \\
\hline$[9]$ & O2I & $2.154 \mathrm{GHz}$ & $30 \mathrm{MHz}$ & SISO & PL, DS \\
\hline$[10]$ & O2I/ & $2.5 \mathrm{GHz}$ & $50 \mathrm{MHz}$ & $\begin{array}{l}\text { MISO/ } \\
\text { SIMO }\end{array}$ & $\begin{array}{l}\text { DS, AoA, } \\
\text { AoD, PAS, DF }\end{array}$ \\
\hline I2O & & N/A & SISO & PL, SF \\
\hline$[11]$ & O2I & $930 \mathrm{MHz}$ & N/A &
\end{tabular}

O2I: outdoor-to-indoor; I2O: indoor-to-outdoor; SISO: single-input singleoutput; MISO: multiple-input single-output; SIMO: single-input, multiple output; PL: path loss; DS: RMS delay spread; $K$ : Ricean $K$-factor; PAS: power azimuth spectrum; DF: Doppler frequency; SF: shadow fading 


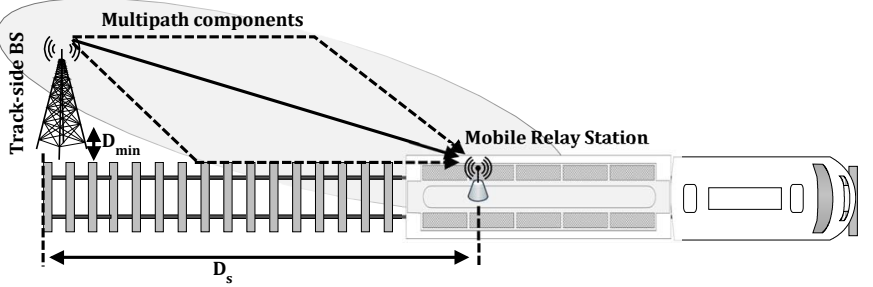

Fig. 1. A HST communication system.

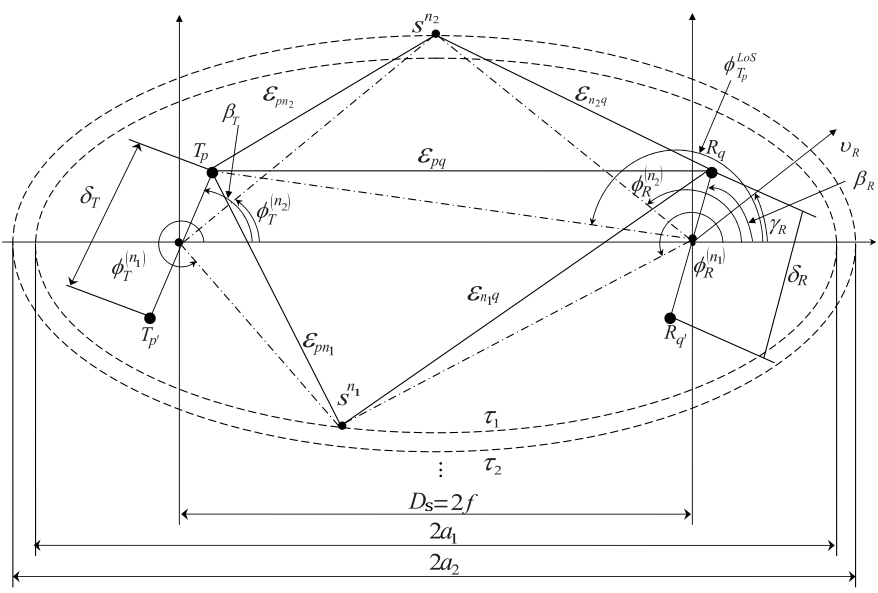

Fig. 2. The GBSM for a MIMO HST channel.

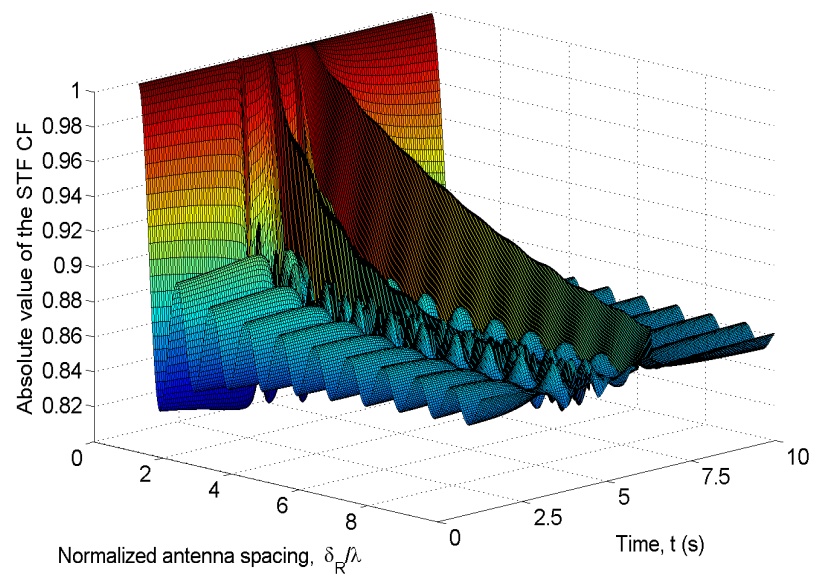

Fig. 3. The absolute values of the non-stationary STF CF of the first tap of the proposed HST channel model.

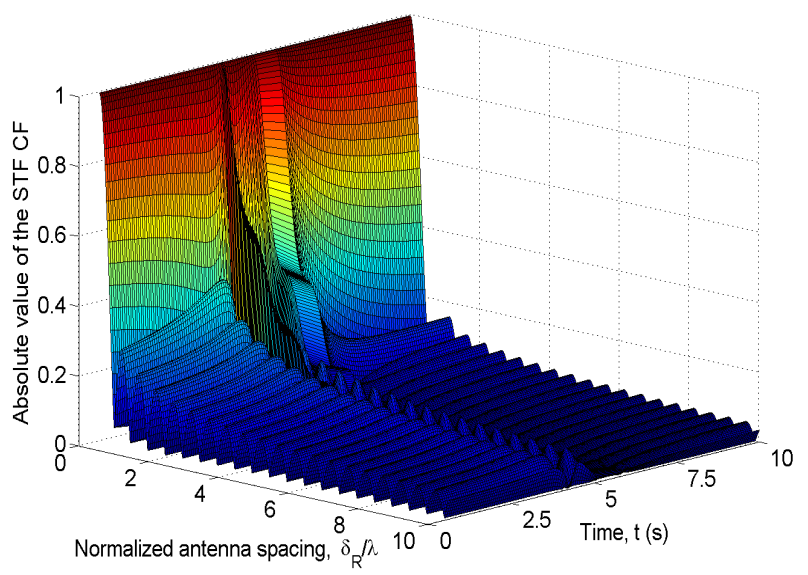

Fig. 4. The absolute values of the non-stationary STF CF of the second tap of the proposed HST channel model.

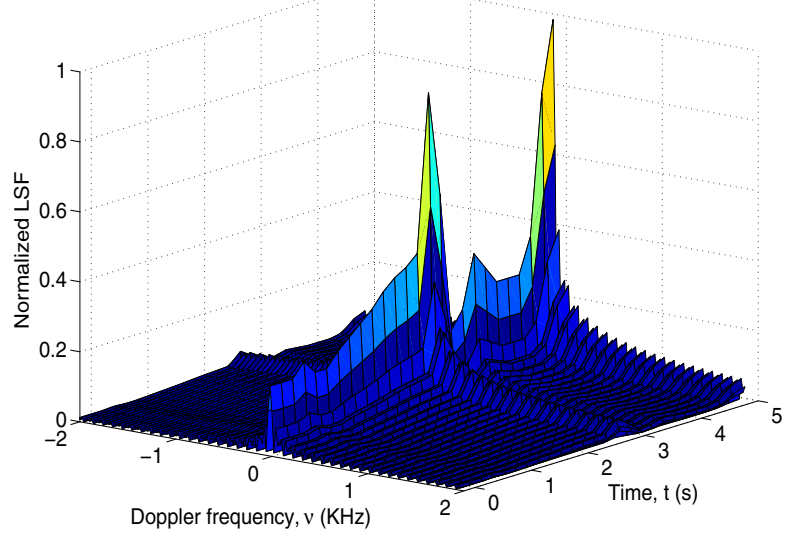

Fig. 5. The absolute values of the non-stationary STF LSF of the second tap of the proposed HST channel model

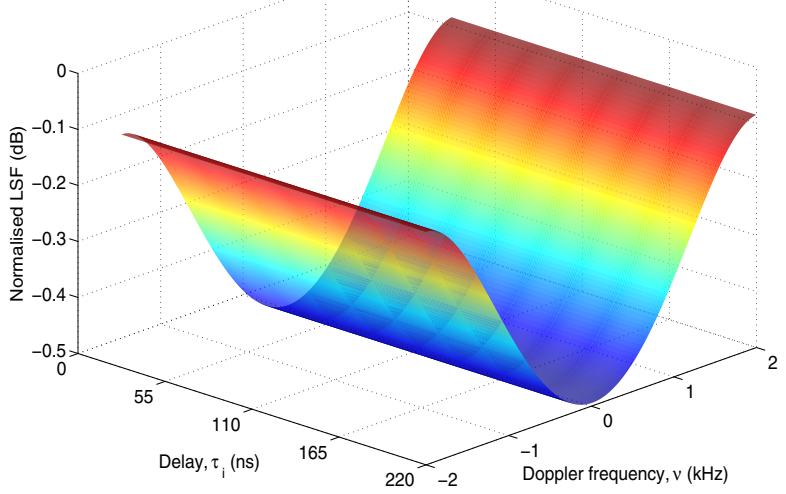

Fig. 6. The normalized STF LSF of the proposed HST channel model of different taps at a certain time $(t=0)$. 